\begin{tabular}{|c|c|}
\hline \multirow{3}{*}{ 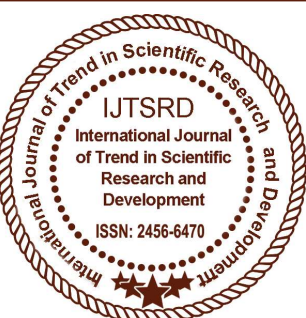 } & $\begin{array}{l}\text { International Journal of Trend in Scientific } \\
\text { Research and Development (IJTSRD) }\end{array}$ \\
\hline & Access Journal \\
\hline & ISSN No: 2456 - 6470 | www.ijtsrd.com | Volume - 2 | Issue -3 \\
\hline
\end{tabular}

\title{
Time Delay and Mean Square Stochastic Differential Equations in Impetuous Stabilization
}

\begin{abstract}
G. Pramila
Department of Mathematics, Vivekanandha College of Arts and Sciences for Women (Autonomous), Elayampalayam, Thiruchengode, Namakkal, Tamil Nadu, India
\end{abstract}

\begin{abstract}
S. Ramadevi
Department of Mathematics, Vivekanandha College of Arts and Sciences for Women (Autonomous), Elayampalayam, Thiruchengode, Namakkal, Tamil Nadu, India
\end{abstract}

\section{ABSTRACT}

This paper specially exhibits about the time delay and mean square stochastic differential equations in impetuous stabilization is analyzed. By projecting a delay differential inequality and using the stochastic analysis technique, a few present stage for mean square exponential stabilization are survived. It is express that an unstable stochastic delay system can be achieved some stability by impetuous. This example is also argued to derived the efficiency of the obtained results.

Keywords: Impetuous stabilization, mean square stochastic delay differential equation, differential inequality

\section{INTRODUCTION}

Impetuous control and stability has been extensively the variety of areas has found many application to be studied over the past centuries, such as orbital transfer of satellite [11], ecosystems management [12,13], dosage supply in pharmacokinetics [14], and stability and synchronization in chaotic secure communication systems and other chaotic systems $[15,16]$. At present, many significant results for the impetuous control and stabilization of delay differential systems.

Systematically, the input states of systems not only get the disturbance of delay phenomena, but also get the disturbance of mean square stochastic phenomena in many practical problems. In current years, the stability investigation of impetuous stochastic delay differential systems is catching to many authors, and a large number of stability criteria of these systems have been reported [1-6]. However, these criteria are all needed so that the corresponding mean square stochastic delay differential systems without impetuous must be stable itself, which implies that the impetuous are only to inhibit the stability of mean square stochastic delay differential systems. Although, it is known that ther is no general rule for guiding how a proper Lyapunov functional can be constructed for a given model. Therefore, it is very impetuous to projecting a new approach to studying the impetuous stabilization of mean square stochastic functional differential equations without resorting to the Lyapunov functional.

Without motivation from the above discussions, our main aim in this paper is to the study the time delay and mean square stochastic differential equations in impetuous stabilization. By projecting a delay differential inequality and using the stochastic analysis technique, some sufficient conditions for mean square exponential stability are obtained.

\section{Model and Preliminaries}

In this paper, unless otherwise specified, we adopt the standard notation used previously by Yang [8]. Let $\mathrm{E}$ denote the $n$-dimensional unit matrix, $|$.$| denote the$ Euclidean norm,

$\mathcal{N} \triangleq\{1,2, \ldots, n\}, \mathbb{N} \triangleq\{1,2, \ldots\}, \mathbb{R}_{+}=[0, \infty), \mathbb{R}_{+}^{0}=$ $(0, \infty)$. for $A \in \mathbb{R}^{n \times n}, \lambda_{\text {max }}(A)$ and $\lambda_{\text {min }}(A)$ are 
largest and smallest singular values of $\mathrm{A}$, respectively. $\|A\|=\sqrt{\lambda_{\max }\left(A^{T} A\right)}$.

$\mathrm{B}[\mathrm{X}, \mathrm{Y}]$ denotes the space of continuous mappings from the topological space $Y$. In particular, let $B \triangleq B\left[[-\tau, 0], \mathbb{R}^{n}\right]$, where $\tau>0$ :

$\mathrm{PB}\left[\mathbb{J}, \mathbb{R}^{\mathrm{n}}\right]=\left\{\psi: \mathbb{J} \rightarrow \mathbb{R}^{\mathrm{n}}\right.$ ।

$\psi(\mathrm{s})$ is continuous for all but at most countable ponit $s \in \mathbb{J}$ and at these points $\in$ $\mathbb{J}, \psi\left(\mathrm{s}^{+}\right)$and $\psi\left(\mathrm{s}^{-}\right)$exist and $\left.\psi(\mathrm{s})=\psi\left(\mathrm{s}^{+}\right)\right\}$,

Where $\mathbb{D} \subset \mathbb{R}$ is an interval, $\psi\left(\mathrm{s}^{+}\right)$and $\psi\left(\mathrm{s}^{-}\right)$denot the right-hand and left-hand limits of function $\psi(\mathrm{s})$ at time $\mathrm{s}$, respectively. In particular, let $P B \triangleq$ $P B\left[[-\tau, 0], \mathbb{R}^{n}\right]:$

$\mathscr{H}=\left\{h(s): \mathbb{R} \rightarrow \mathbb{R}_{+}^{0} \mid\right.$

$h(s)$ is integral and satisfies $\sup _{t \geq t_{0}} \int_{t-\tau}^{t} h(s) d s=$ $H<\infty$, and $\lim _{t \rightarrow \infty} \int_{t_{0}}^{t} h(s) d s=$

$\infty$, where $H$ is a positive constant $\}$.

For $\varphi \in B\left[\mathbb{J}, \mathbb{R}^{\mathrm{n}}\right]$ or $\varphi \in P B\left[\mathbb{J}, \mathbb{R}^{\mathrm{n}}\right]$, we define $[\varphi(t)]_{\tau}=\left(\left[\varphi_{1}(t)\right]_{\tau}, \ldots,\left[\varphi_{n}(t)\right]_{\tau}\right)^{T}$, $\left[\varphi_{i}(t)\right]_{\tau}=\sup _{-\tau \leq s \leq 0}\left\{\varphi_{i}(t+s)\right\}, i \in \mathcal{N}$, And $D^{+} \varphi(t)$ denotes the upper right-hand derivative of $\varphi(t)$ at time t.

Let $\left(\Omega, \mathscr{F}_{,}\left\{\mathscr{F}_{t}\right\}_{t \geq 0}, P\right)$ be a complete probability space with a filtration $\left\{\mathscr{F}_{t}\right\}_{t \geq 0}$ satisfying the usual conditions (i.e., it is right continuous and $\mathscr{F}_{0}$ contains all p-null sets ). $N_{\text {Let }}$ $P B_{\mathscr{F}_{0}}^{b}\left[[-\tau, 0], \mathbb{R}^{n}\right]\left(P B_{\mathscr{F}_{t}}^{b}\left[[-\tau, 0], \mathbb{R}^{n}\right]\right)$ denote the family of all bounded $\mathscr{F}_{0}\left(\mathscr{F}_{t}\right)$-measurable, $P B\left[[-\tau, 0], \mathbb{R}^{n}\right]$-valued random variable $\phi$, satisfying $\|\phi\|_{L^{2}}^{2}=\sup _{-\tau \leq \theta \leq 0} \mathbb{E}|\phi(\theta)|^{2}<\infty$, where $\mathbb{E}$ denotes the expectation of stochastic process. Let $\mathscr{L}$ denote the well-known $\mathscr{L}$-operator given by the Ito formula.

In this paper, we consider the following Ito impetuous stochastic delay systems:

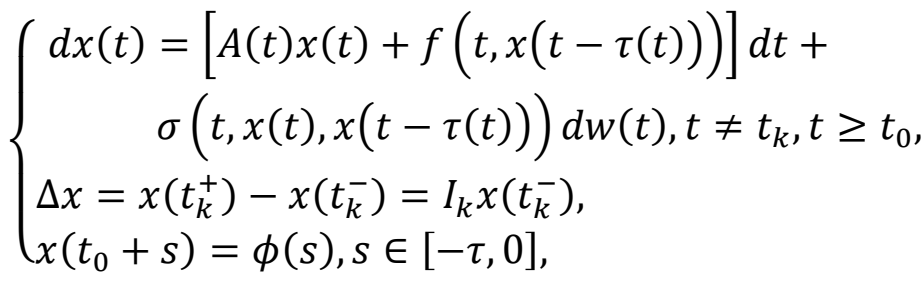

$A(t)=h(t) A, h(t) \in \mathscr{H}, A \in \mathbb{R}^{n \times n} . I_{k} \in \mathbb{R}^{n \times n}, 0 \leq$ $\tau(t) \leq \tau, \tau$ is a constant, and fixed impetuous moment $t_{k}(k \in \mathbb{N})$ satisfy $t_{1}<t_{2}<\cdots \quad$ and $\lim _{k \rightarrow \infty} t_{k}=\infty . \quad f: \mathbb{R}_{+} \times \mathbb{R}^{n} \rightarrow \mathbb{R}^{n}$ and $\sigma: \mathbb{R}_{+} \times \mathbb{R}^{n} \times$ $\mathbb{R}^{n} \rightarrow \mathbb{R}^{n \times m}$ are piecewise continuous vector-valued functions with $f(t, 0)=\sigma(t, 0,0) \equiv 0, t \in \mathbb{R}_{+}$, and ensuring the existence and uniqueness of (1). One may refer to[9,10] for the results on the existence and uniqueness of solutions of impetuous stochastic system.

The solution $x(t)=\left(x_{1}(t), \ldots, x_{n}(t)\right)^{T}$ is a PB valued stochastic process.

$w(t)=\left(w_{1}(t), \ldots, w_{m}(t)\right)^{T}$ is an m-dimensional

Brownian motion defined $\left.\left(\Omega, \mathscr{F}_{,}, \mathscr{F}_{t}\right\}_{t \geq 0}, P\right)$.

\section{Definition 2.1}

The trivial solution of (1) is said to be globally mean square asymptotically stable if for any given initial condition $\phi \in P B_{\mathscr{Y}_{0}}^{b}\left[[-\tau, 0], \mathbb{R}^{n}\right]$ such that

$$
\lim _{t \rightarrow \infty} \mathbb{E}\left|x\left(t, t_{0}, \phi\right)\right|^{2}=0 \text {. }
$$

\section{Definition 2.2}

The trivial solution of (1) is said to be globally mean square exponentially stable if there exist constants $\lambda>0$ and $k>1$ such that for any solution $\mathrm{x}(\mathrm{t})$ with the initial condition $\phi \in P B_{\mathscr{F}_{0}}^{b}\left[[-\tau, 0], \mathbb{R}^{n}\right]$,

$$
\mathbb{E}|x(t)|^{2} \leq k\|\phi\|_{L^{2}}^{2} e^{-\lambda\left(t-t_{0}\right)}, \quad t \geq t_{0} .
$$

\section{Main results}

In this section, we will first projecting an impetuous differential inequality with delays and then investigate the mean square stability of (1) by employing the obtained impetuous differential inequality, and stochastic analysis technique. It is shown that an unstable stochastic delays system can be successfully stabilized by impetuous.

\section{Theorem 3.1}

Let $q \geq 0$, and $X(t), Y(t) \in P B\left[\left[t_{0}, \infty\right), \mathbb{R}\right]$ be a solution of the following impetuous delay differential inequality with the initial condition $X(t), Y(t) \in$ $P B,-\tau \leq s \leq t_{0}$,

Where the initial function $\phi(s)=\left(\phi_{1}(s), \ldots, \phi_{n}(s)\right)^{T} \in P B_{\mathscr{F}_{t}}^{b}\left[[-\tau, 0], \mathbb{R}^{n}\right]$. 
$\left\{\begin{array}{l}D^{+} X(t) \leq h(t)\left[-P X(t)+q[X(t)]_{\tau}\right], \quad t \neq t_{k}, t \geq t_{0} \\ X\left(t_{k}\right) \leq \alpha_{k} X\left(\bar{t}_{k}\right), \quad 0<\alpha_{k-1}<1, k \in \mathbb{N}, \\ X\left(t_{0}+s\right)=\phi(s) \in P B[[-\tau, 0], \mathbb{R}] \quad-\tau \leq s \leq 0\end{array}\right.$
+
$\left\{\begin{array}{l}D^{+} Y(t) \leq h(t)\left[-P Y(t)+q[Y(t)]_{\tau}\right], \quad t \neq t_{n}, t \geq t_{0} \\ Y\left(t_{n}\right) \leq \alpha_{n} Y\left(\bar{t}_{n}\right), \quad 0<\alpha_{n-1}<1, n \in \mathbb{N}, \\ Y\left(t_{0}+s\right)=\phi(s) \in P B[[-\tau, 0], \mathbb{R}] \quad-\tau \leq s \leq 0\end{array}\right.$

Where $h(t) \in \mathscr{H}$. Assume that the following condition is satisfied

$$
\begin{aligned}
& \left(-p+\frac{q}{\alpha_{k-1}}\right)\left(\int_{t_{k-1}}^{t_{k}} h(s) d s\right)+ \\
& \left(-p+\frac{q}{\alpha_{n-1}}\right)\left(\int_{t_{n-1}}^{t_{n}} h(s) d s\right)<-\operatorname{In} \alpha_{k-1}-\operatorname{In} \alpha_{n-1}
\end{aligned}
$$

$k \in \mathbb{N}$.

Then there exist a constant $M \geq 1, G \geq 1$ and a small enough number $\lambda>0, \mu>0$ such that

$X(t)+Y(t) \leq$

$\|\phi\| M e^{-\lambda \int_{t_{0}}^{t} h(s) d s}+\|\phi\| G e^{-\mu \int_{t_{0}}^{t} h(s) d s}, t \geq t_{0}$

Where $\|\phi\|=\sup _{-\tau \leq s \leq 0}|\phi(s)|$, and the constants $\lambda>0, \mu>0$ satisfies the following inequality

$\left(2 \lambda-p+\frac{q e^{\lambda H}}{\alpha_{k-1}}\right)\left(\int_{t_{k-1}}^{t_{k}} h(s) d s\right)+(2 \mu-p+$

$\left.\frac{q e^{\mu H}}{\alpha_{n-1}}\right)\left(\int_{t_{n-1}}^{t_{n}} h(s) d s\right)<-\operatorname{In} \alpha_{k-1}-\operatorname{In} \alpha_{n-1}, k \in$

$\mathbb{N}, n \in \mathbb{N}$.

\section{Proof}

We shall show that

$X(t)+Y(t) \leq$

$\|\phi\| M e^{-\lambda \int_{t_{0}}^{t} h(s) d s}+\|\phi\| G e^{-\mu \int_{t_{0}}^{t} h(s) d s}, t \in$

$\left[t_{k-1}, t_{k}\right), k \in \mathbb{N} \& t \in\left[t_{n-1}, t_{n}\right), n \in$

$\mathbb{N}$.

By using the continuity, from (3) we know that there must there must exist a positive constants $\lambda$ and $\mu$ such that for all (5) holds.

$$
\text { Let } \gamma=\sup _{k \in \mathbb{N}}\left\{\frac{1}{\alpha_{k-1}}\right\} \geq 1 \text { and } \delta=
$$

$\sup _{n \in \mathbb{N}}\left\{\frac{1}{\alpha_{n-1}}\right\} \geq 1$ in (5), then we can select constant $\eta>0, \rho>0$ such that for all $k \in \mathbb{N}, n \in \mathbb{N}$.
$-2 p+\gamma q e^{\lambda H}+\delta q e^{\mu H}<-\operatorname{In} \alpha_{k-1}-\operatorname{In} \alpha_{n-1}, k \in$

$\mathbb{N}, n \in \mathbb{N}$.

And

$$
(\eta+\lambda)\left(\int_{t_{k-1}}^{t_{k}} h(s) d s\right)+(\rho+\mu)\left(\int_{t_{n-1}}^{t_{n}} h(s) d s\right)<
$$

$-\operatorname{In} \alpha_{k-1}-\operatorname{In} \alpha_{n-1} \leq \operatorname{In} \gamma+\operatorname{In} \delta$

From (8) we can choose $M \geq 1, G \geq 1$ such that

$1<e^{(\eta+\lambda) \int_{t_{0}}^{t_{1}} h(s) d s}+e^{(\rho+\mu) \int_{t_{0}}^{t_{1}} h(s) d s} \leq M+G \leq$ $\gamma e^{\lambda H-(\eta+\lambda) \int_{t_{0}}^{t_{1}} h(s) d s} e^{(\eta+\lambda) \int_{t_{0}}^{t_{1}} h(s) d s}+$

$\delta e^{\mu H-(\rho+\mu) \int_{t_{0}}^{t_{1}} h(s) d s} e^{(\rho+\mu) \int_{t_{0}}^{t_{1}} h(s) d s}$.

It then follows that

$$
\begin{aligned}
& \leq\|\phi\| M e^{-\lambda \int_{t_{0}}^{t_{1}} h(s) d s} \\
& +\|\phi\| G e^{-\mu \int_{t_{0}}^{t_{1}} h(s) d s}
\end{aligned}
$$

We first prove that

$X(t)+Y(t) \leq$

$\|\phi\| M e^{-\lambda \int_{t_{0}}^{t} h(s) d s}+\|\phi\| G e^{-\mu \int_{t_{0}}^{t} h(s) d s}, t \in$

$\left[t_{0}, t_{1}\right)$.

To do this, we only to prove that

$$
\begin{aligned}
& X(t)+Y(t) \\
& \leq\|\phi\| M e^{-\lambda \int_{t_{0}}^{t_{1}} h(s) d s} \\
& +\|\phi\| G e^{-\mu \int_{t_{0}}^{t_{1}} h(s) d s}
\end{aligned}
$$

If (12) is not true, by (10), then there exists some $\bar{t} \in\left(t_{0}, t_{1}\right)$ such that

$$
\begin{aligned}
X(\bar{t})+Y(\bar{t})> & \|\phi\| M e^{-\lambda \int_{t_{0}}^{t_{1}} h(s) d s} \\
& +\|\phi\| G e^{-\mu \int_{t_{0}}^{t_{1}} h(s) d s} \\
& \geq\|\phi\| e^{\eta \int_{t_{0}}^{t_{1}} h(s) d s}+\|\phi\| e^{\rho \int_{t_{0}}^{t_{1}} h(s) d s} \\
& >\|\phi\| \geq u\left(t_{0}+s\right), \quad s \in[-\tau, 0]
\end{aligned}
$$

Which implies that there exists some $t^{*} \in\left(t_{0}, \bar{t}\right)$ such that

$X\left(t^{*}\right)+Y\left(t^{*}\right)=$

$\|\phi\| M e^{-\lambda \int_{t_{0}}^{t_{1}} h(s) d s}+\|\phi\| G e^{-\mu \int_{t_{0}}^{t_{1}} h(s) d s}$,

and $X(t)+Y(t) \leq X\left(t^{*}\right)+Y\left(t^{*}\right), \quad t \in\left[t_{0}-\tau, t^{*}\right]$ (14) 
And there exists $t^{* *} \in\left[t_{0}, t^{*}\right)$ such that

$X\left(t^{* *}\right)+Y\left(t^{* *}\right)=\|\phi\|, \quad$ and $X\left(t^{* *}\right)+Y\left(t^{* *}\right) \leq$ $X(t)+Y(t) \leq X\left(t^{*}\right)+Y\left(t^{*}\right), \quad t \in\left[t^{* *}, t^{*}\right]$.

Hence, we get for any $s \in[-\tau, 0]$,

$$
\begin{aligned}
& X(t+s)+Y(t+s) \\
& \leq\|\phi\| M e^{-\lambda \int_{t_{0}}^{t_{1}} h(s) d s}+\|\phi\| G e^{-\mu \int_{t_{0}}^{t_{1}} h(s) d s} \\
& \leq\|\phi\| \gamma e^{\lambda H-(\eta+\lambda) \int_{t_{0}}^{t_{1}} h(s) d s} e^{(\eta+\lambda) \int_{t_{0}}^{t_{1}} h(s) d s} \\
& +\|\phi\| \delta e^{\mu H-(\rho+\mu) \int_{t_{0}}^{t_{1}} h(s) d s} e^{(\rho+\mu) \int_{t_{0}}^{t_{1}} h(s) d s} \\
& =\gamma e^{\lambda H} X\left(t^{* *}\right)+\delta e^{\mu H} Y \leq \gamma e^{\lambda H} X(t)+\delta e^{\mu H} Y(t), \\
& t \in\left[t^{* *}, t^{*}\right] .
\end{aligned}
$$

Thus, by (7) and (16), we have

$D^{+} X(t) \leq h(t)\left[-p X(t)+q[X(t)]_{\tau}\right] \leq h(t)[-p+$ $\left.\gamma q e^{\lambda H}\right] X(t) \leq h(t)(\eta-\lambda) X(t), t \in\left[t^{* *}, t^{*}\right]+$ $D^{+} Y(t) \leq h(t)\left[-p Y(t)+q[Y(t)]_{\tau}\right]$

$\leq h(t)\left[-p+\delta q e^{\mu H}\right] Y(t) \leq h(t)(\rho-\mu) Y(t), t \in$ $\left[t^{* *}, t^{*}\right]$

It follows from (10),(14) and (15) that

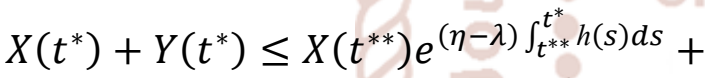
$Y\left(t^{* *}\right) e^{(\rho-\mu) \int_{t^{*}}^{t^{*}} h(s) d s}<\|\phi\| e^{\eta \int_{t_{0}}^{t_{1}} h(s) d s}+$ $\|\phi\| e^{\rho \int_{t_{0}}^{t_{1}} h(s) d s} \leq\|\phi\| M e^{-\lambda \int_{t_{0}}^{t_{1}} h(s) d s}+$ $\|\phi\| G e^{-\mu \int_{t_{0}}^{t_{1}} h(s) d s}=X\left(t^{*}\right)+Y\left(t^{*}\right)$

Which is contradiction. Hence (12) holds and then (6) is true for $k=1$. Now we assume that (6) holds for $k=1,2, \ldots, m(m \in \mathbb{N}, m \geq 1)$,

$X(t)+Y(t) \leq$

$\|\phi\| M e^{-\lambda \int_{t_{0}}^{t} h(s) d s}+\|\phi\| G e^{-\mu \int_{t_{0}}^{t} h(s) d s}, t \in$ $\left[t_{k-1}, t_{k}\right)$,

$k=1,2, \ldots, m$.

Next, To find equation (6) holds for $k=m+1$,

$(t)+Y(t) \leq$

$\|\phi\| M e^{-\lambda \int_{t_{0}}^{t} h(s) d s}+\|\phi\| G e^{-\mu \int_{t_{0}}^{t} h(s) d s}, \quad t \in$ $\left[t_{m}, t_{m+1}\right)$.
For the reasons of contradiction, suppose (20) is not true. Then we define

$\bar{t}=$

$\inf \left\{t \in\left[t_{m}, t_{m+1}\right) \mid X(t)+Y(t)>\right.$ $\left.\|\phi\| M e^{-\lambda \int_{t_{0}}^{t} h(s) d s}+\|\phi\| G e^{-\mu \int_{t_{0}}^{t} h(s) d s}\right\}$.

By (8) and (19), we have

$$
\begin{aligned}
& X\left(t_{m}\right)+Y\left(t_{m}\right) \\
& \leq \alpha_{m}\|\phi\| M e^{-\lambda \int_{t_{0}}^{t_{m}} h(s) d s}+\alpha_{m}\|\phi\| G e^{-\mu \int_{t_{0}}^{t_{m}} h(s) d s} \\
& <\alpha_{m} e^{\lambda \int_{t_{m}}^{t_{m+1}} h(s) d s}\|\phi\| M e^{-\lambda \int_{t_{0}}^{t} h(s) d s} \\
& +\alpha_{m} e^{\mu \int_{t_{m}}^{t_{m+1}} h(s) d s}\|\phi\| G e^{-\mu \int_{t_{0}}^{t} h(s) d s} \\
& <\|\phi\| M e^{-\lambda \int_{t_{0}}^{t} h(s) d s} \\
& +\|\phi\| G e^{-\mu \int_{t_{0}}^{t} h(s) d s}
\end{aligned}
$$

And so $\bar{t} \neq t_{m}$. By utilizing the continuity of $X(t)+Y(t)$ in the interval $\left[t_{m}, t_{m+1}\right)$, we get

$$
X(\bar{t})+Y(\bar{t})=
$$

$\|\phi\| M e^{-\lambda \int_{t_{0}}^{t} h(s) d s}+\|\phi\| G e^{-\mu \int_{t_{0}}^{\bar{t}} h(s) d s}$, and $X(t)+$

$Y(t) \leq X(\bar{t})+Y(\bar{t}), t \in\left[t_{m}, \bar{t}\right]$.

From (22), we know that there exist some $t^{*} \in\left(t_{m}, \bar{t}\right)$ such that

$$
\begin{aligned}
& X\left(t^{*}\right)+Y\left(t^{*}\right)= \\
& \alpha_{m} e^{\lambda \int_{t_{m}}^{t_{m+1}} h(s) d s}\|\phi\| M e^{-\lambda \int_{t_{0}}^{t} h(s) d s}+ \\
& \alpha_{m} e^{\mu \int_{t_{m}}^{t_{m+1}} h(s) d s}\|\phi\| G e^{-\mu \int_{t_{0}}^{t} h(s) d s},
\end{aligned}
$$

$$
\text { and } X\left(t^{*}\right)+Y\left(t^{*}\right) \leq X(t)+Y(t) \leq
$$

$X(\bar{t})+Y(\bar{t}), \quad t \in\left[t_{m}, \bar{t}\right]$.

On the other hand, for any $t \in\left[t^{*}, \bar{t}\right], s \in$ $[-\tau, 0]$, either $t+s \in\left[t_{0}-\tau, t_{m}\right)$ or $t+s \in\left[t_{m}, \bar{t}\right]$. Two cases will be discussed as follows. If $t+s \in$ $\left[t_{0}-\tau, t_{m}\right)$, from (19),we obtain $X(t+s)+$ $Y(t+s) \leq\|\phi\| M e^{-\lambda \int_{t_{0}}^{t} h(s) d s} e^{-\lambda \int_{t}^{t+s} h(s) d s}+$ $\|\phi\| G e^{-\mu \int_{t_{0}}^{t} h(s) d s} e^{-\mu \int_{t}^{t+s} h(s) d s} \leq$

$$
\|\phi\| M e^{-\lambda \int_{t_{0}}^{t} h(s) d s} e^{\lambda H}+
$$

$\|\phi\| G e^{-\mu \int_{t_{0}}^{t} h(s) d s} e^{\mu H}$

$$
\leq\|\phi\| e^{\lambda H} e^{\lambda \int_{t_{m}}^{t_{m+1}} h(s) d s} M e^{-\lambda \int_{t_{0}}^{\bar{t}} h(s) d s}+
$$


$\|\phi\| e^{\mu H} e^{\mu \int_{t_{m}}^{t_{m}+1} h(s) d s} G e^{-\mu \int_{t_{0}}^{\bar{t}} h(s) d s}$

if $t+s \in\left[t_{m}, \bar{t}\right]$, form (23), then

$X(t+s)+Y(t+s)=\|\phi\| M e^{-\lambda \int_{t_{0}}^{\bar{t}} h(s) d s}+$

$\|\phi\| G e^{-\mu \int_{t_{0}}^{\bar{t}} h(s) d s} \leq$

$\|\phi\| e^{\lambda H} e^{\lambda \int_{t_{m}}^{t_{m+1}} h(s) d s} M e^{-\lambda \int_{t_{0}}^{\bar{t}} h(s) d s}+$

$\|\phi\| e^{\mu H} e^{\mu \int_{t_{m}}^{t_{m}} h(s) d s} G e^{-\mu \int_{t_{0}}^{\bar{t}} h(s) d s}$

In any case, from (24)-(26), we all have for any $s \in[-\tau, 0]$,

$c[X(t+s)+Y(t+s)] \leq \frac{e^{\lambda H}}{\alpha_{m}} X\left(t^{*}\right)+\frac{e^{\mu H}}{\alpha_{m}} Y\left(t^{*}\right) \leq$ $\gamma e^{\lambda H} X(t)+\delta e^{\mu H} Y(t), t \in\left[t^{*}, \bar{t}\right]$,

Finally, by (7) and (27), we all have

$D^{+}[X(t)+Y(t)] \leq h(t)[(\eta-\lambda) X(t)+$

$\rho-\mu Y t$.

It follows from (8), (23) and (24) that

$$
\begin{aligned}
& X(\bar{t})+Y(\bar{t}) \\
& \leq X\left(t^{*}\right) e^{(\eta-\lambda) \int_{t^{*}}^{\bar{t}} h(s) d s} \\
& +Y\left(t^{*}\right) e^{(\rho-\mu) \int_{t^{*}}^{\bar{t}} h(s) d s} \\
& <e^{-(\eta+\lambda) \int_{t_{m}}^{t_{m}+1} h(s) d s} e^{\lambda \int_{t_{m}}^{t_{m}+1} h(s) d s}\|\phi\| M e^{-\lambda \int_{t_{0}}^{\bar{t}} h(s) d s} \\
& +e^{-(\rho+\mu) \int_{t_{m}}^{t_{m}+1} h(s) d s} e^{\mu \int_{t_{m}}^{t_{m}} h(s) d s}\|\phi\| G \\
& <\|\phi\| M e^{-\lambda \int_{t_{0}}^{\bar{t}} h(s) d s}+\|\phi\| G e^{-\mu \int_{t_{0}}^{\bar{t}} h(s) d s} \\
& \quad=X(\bar{t})+Y(\bar{t})
\end{aligned}
$$

Which is a contradiction. This implies that the assumption is not true, and hence (6) holds for $k=m+1$. Therefore, by some simple mathematical induction, we can obtain that (6) holds for any $k \in \mathbb{N}$. This completes the proof of theorem.

\section{References}

1) Z.G. Yang, D.Y.Xu, L.Xiang, Exponential pstability of impulsive stochastic differential equations with delays, Phys. Lett. A 359 (2006) 129-137.

2) L.G.Xu, D.Y.Xu, p-attracting and p-invariant sets for a class of impulsive stochastic functional differential equations, comput. Math. Appl. 57 (2009) 54-61.
3) L.G.Xu, D.Y.Xu, Exponential p-stability of impulsive stochastic neural networks with mixed delays, Chaos Solitons Fractals 41(2009) 263272.

4) L.G.Xu, Exponential p-stability of singularly perturbed impulsive stochastic delay differential systems, Int. J. Control Autom. 9 (2011) 966-972.

5) L.G.Xu, D.H.He, Mean square exponential stability analysis of impulsive stochastic switched (28ystems with mixed delays, comput. Math. Appl. 62 (2011) 109-117.

6) J.Liu, X.Z.Liu, W.C.Xie, Impulsive stabilization of stochastic functional differential equations, Appl. Math. Lett. 24 (2011) 264-269.

7) J.Zhou, Q.J.Wu, L.Xiang, et al., Impulsive synchronization seeking in general complex delayed dynamical networks, Nonlinear Anal. Hybrid Sys. 5 (2011) 513-524.

8) Z.G. Yang, Z.C. Yang, Dissipativity in mean square of non- autonomous impulsive stochastic neural networks with delays, Lecture Notes in Comput. Sci. 6063 (2010) 735-744.

9) B.Liu, X.Z.Liu, X.X.Liao, Existence and uniqueness and stability of solutions for stochastic impulsive systems, J.Syst. Sci. Complex. 20 (2007) 149-158.

10) M.S.Alwan, X.Z.Liu, W.Xie, Existence, continuation and uniqueness problems of stochastic impulsive systems with time delay, J.Franklin Inst. 347 (2010) 1317-1333.

11) X.Z.Liu, A.R.Willms, impulsive controllability of linear dynamical systems with applications to maneuvers of spacecraft, Math. Probl. Eng. 2 (1996) 277-299.

12) D.Bainov, P.S.Simeonov, Systems with impulse Effect: Stability, Theory and Applications, Ellis Horwood Limited, Chichester, 1989.

13) X.Z.Liu, K.Rohlf, Impulsive control of a LotkaVolterra system, IMAJ. Math. Control Inform. 15 (1998) 269-284.

14) V.Lakshmikantham, D.D.Bainov, P.S.Simeonov, Theory of Impulsive Differential Equations, World Scientific, Singapore, 1989.

15) A. Khadra, X.Z.Liu, X.M.Shen, Application of impulsive synchronization to communication security, IEEE Trans. Circuits Syst. I 50 (2003) 341-351.

16) J.T.Sun, Impulsive control of a new chaotic system, Math. Comput. Simul. 64 (2004) 669-677. 